\title{
Detection of Steel Bars in Concrete by Impact-Echo
}

\author{
Z. Hlaváć* \& O. Anton \\ Department of Building Testing, Faculty of Civil Engineering, Brno University of Technology, Brno, Czech \\ Republic, \\ *Corresponding author: zbynek.hlavac@seznam.cz
}

\author{
A. Garbacz \\ Department of Structure Materials, Faculty of Civil Engineering, Warsaw University of Technology, Warsaw, \\ Poland
}

\begin{abstract}
Due to the impact-echo defectoscopy tests of a massive block of underreinforced concrete steel bars of diameter 22 and $32 \mathrm{~mm}$ were detected. This fact is described in the paragraph, and results are provided through a visualization of $b$-scanning. The effect of a concrete cover layer and of the reinforcement diameter is clear on the sensitivity of the method. Because of various parameters of the reinforcement, impactors of a different ball diameter were employed. With a ball of diameter $12 \mathrm{~mm}, 8 \mathrm{~mm}$ and $5 \mathrm{~mm}$ reinforcement at the depth of about 50 to $60 \mathrm{~mm}$ was detected.
\end{abstract}

KEY WORDS: Impact-echo, concrete, non-destructive testing of steel bar detection.

\section{INTRODUCTION}

Concrete structure condition can be deduced from destructive and non-destructive testing (NDT). There is no standard definition for nondestructive testing (Carino, 1999). In general, tests that do not alter the concrete are considered as NDT methods. NDT methods can be used to detect various types of defects like voids, honeycombing, delaminations, cracks, lack of sub-base support, etc. Some instrumental methods are helpful in integrity evaluation:

- stress wave propagation methods (acoustic methods, IE, ultrasonic methods)

- infrared thermography

- ground penetrating radar (GPR), called also radar

The techniques for flaw detection are generally based on the following simple principle: the presence of an internal anomaly interferes with the propagation of certain type of waves. The presence of the anomaly can be inferred by monitoring the response of the test object when it is subjected to these waves. Impact-Echo Method (I-E) is treated as the one of the most promising NDT techniques for the assessment of concrete structures. 


\section{PRINCIPLE OF IMPACT-ECHO METHOD}

Impact-echo is a method for the non-destructive evaluation of concrete, based on the use of an elastic, low energy impact on the surface generating low frequency stress waves (mainly below $60 \mathrm{kHz}$ ). These waves consist of compression (P), shear (S) and surface Rayleigh (R) components which propagate through the structure and are reflected by interfaces within the material or external boundaries. I-E method is very often used for the quality assessment of concrete structures in the following purposes:

- estimation of member thickness from one side

- detection of internal flaws such as voids, delaminations, honeycombing

- estimation of the depth of surface-opening cracks

- evaluation of multilayer system quality

a)

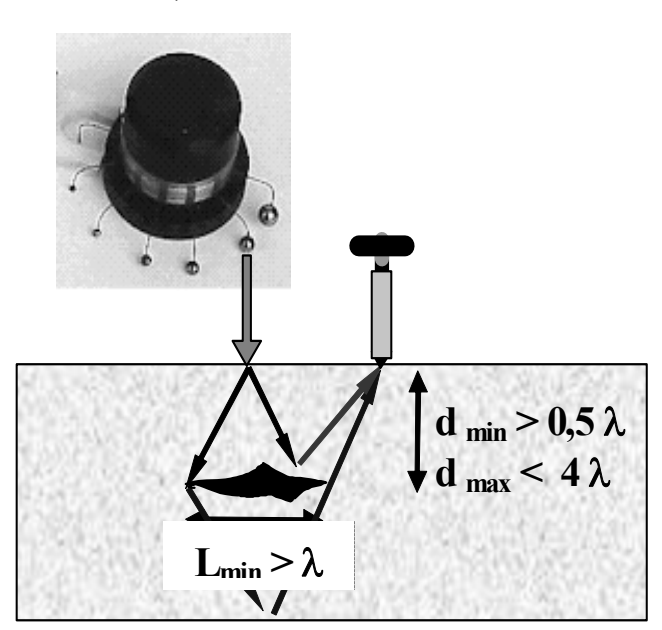

c)

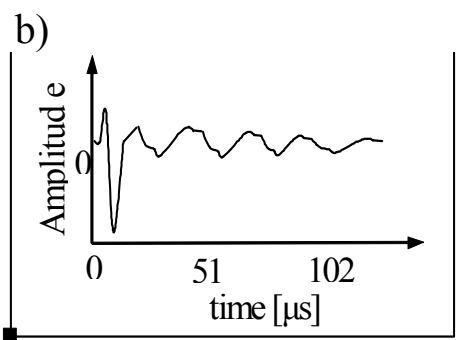

Fast Fourier Transform

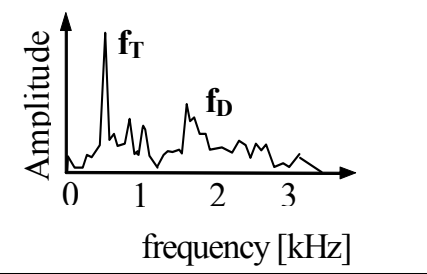

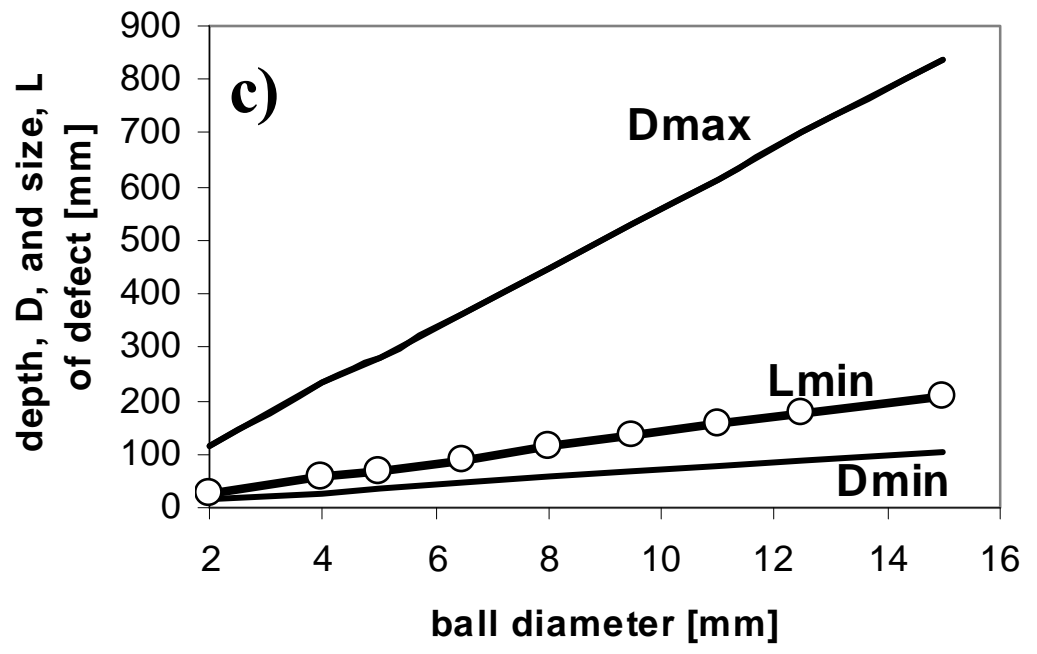

Figure 1: Scheme of impact-echo method a), examples of waveform and corresponding frequency spectrum; b) and c) effect of ball size on depth and size of defect possible to detect 
As the stress waves generated in the I-E method (Fig.1a) have low frequencies (in comparison to, for example, ultrasonic waves) this method is less sensitive to natural heterogeneity of concrete. An additional feature of the I-E method is an application of frequency analysis besides a time-domain analysis (Fig 1b). The selection of the impact source is an important factor for defect detection. The impact duration determines the frequency content of the stress wave and determines the minimum flaw depth that can be detected (Fig.1c). Please note that according to Fig. $3 \mathrm{~b}$ it is possible to detect a steel bar of even half the minimal dimension (diameter) $\mathrm{L}_{\min }$ and cover $\mathrm{D}_{\min }$ in comparison to the defect as delamination or void mentioned in Fig 3c.

On the basis of the frequency spectrum the depth of the reflecting steel interface can be determined according to the relationship (1),

$$
D=\beta C_{p} / 4 f
$$

where: $D$ - depth of interface, $C_{\mathrm{p}}-\mathrm{P}$-wave velocity, $f$ - frequency of dominant peak in frequency spectrum, $\beta$ is a "shape factor" that depends on the geometry of the structure being tested, and the key frequency — called the "thickness frequency" - is the vibration frequency induced by multiple P-wave reflections between the top and bottom surfaces. The shape factor $\beta=0.96$ is valid for a plate structure.

In figure 2 there is scheme of stress wave propagation. In figure 3 the frequency spectra by testing of steel reinforced concrete is shown.
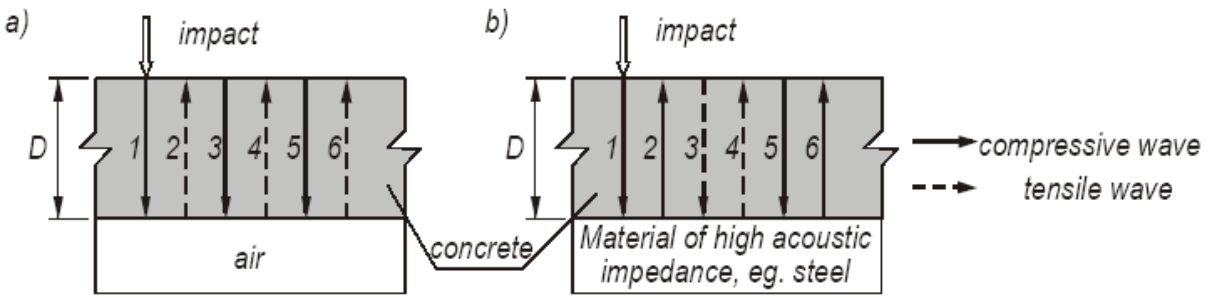

Figure 2: Scheme of stress wave propagation: a) concrete/air and b) concrete/ steel material with higher stiffness (Sansalone \& Street, 1997)

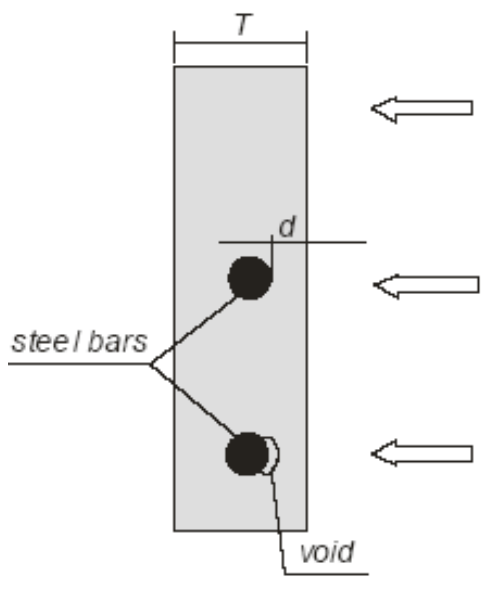

a)

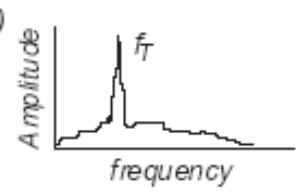

b)

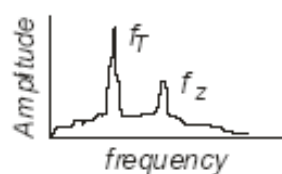

c)

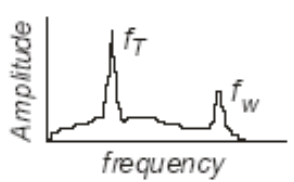

$$
f_{T}=\frac{c_{p}}{2 T}
$$$$
f_{z}=\frac{c_{p}}{4 d}
$$$$
f V=\frac{c_{p}}{2 d}
$$

Figure 3: Scheme of steel bar localization with impact-echo method: a) place without reinforcement $b$ ) place with reinforcement, c) air void at the interface bar/concrete (Runkiewicz, 2002) 


\section{SPECIFICATION OF TESTED AREA}

The tested concrete block is located in the yard of the Civil Engineering Faculty of Brno University of Technology, it has a complex shape (Fig.4a) - from the point of view of impact-echo testing - a plate structure is preferable in this method. The layout dimensions are $1.8 \times 1.8 \mathrm{~m}$, the height of the block is $1.5 \mathrm{~m}$. Measurements were done in the front wall of the block (Fig.4b) where all the implemented defects were located, in the points of previous ultrasonic testing. The point symbols of the ultrasonic grid, $10 \times 10 \mathrm{~cm}$, were used in the I-E analysis. Nevertheless the grid of I-E testing points was $10 \times 20 \mathrm{~cm}-$ more frequent in horizontal direction. The tests were performed with DOCter impact-echo system produced by German Instruments.

a)

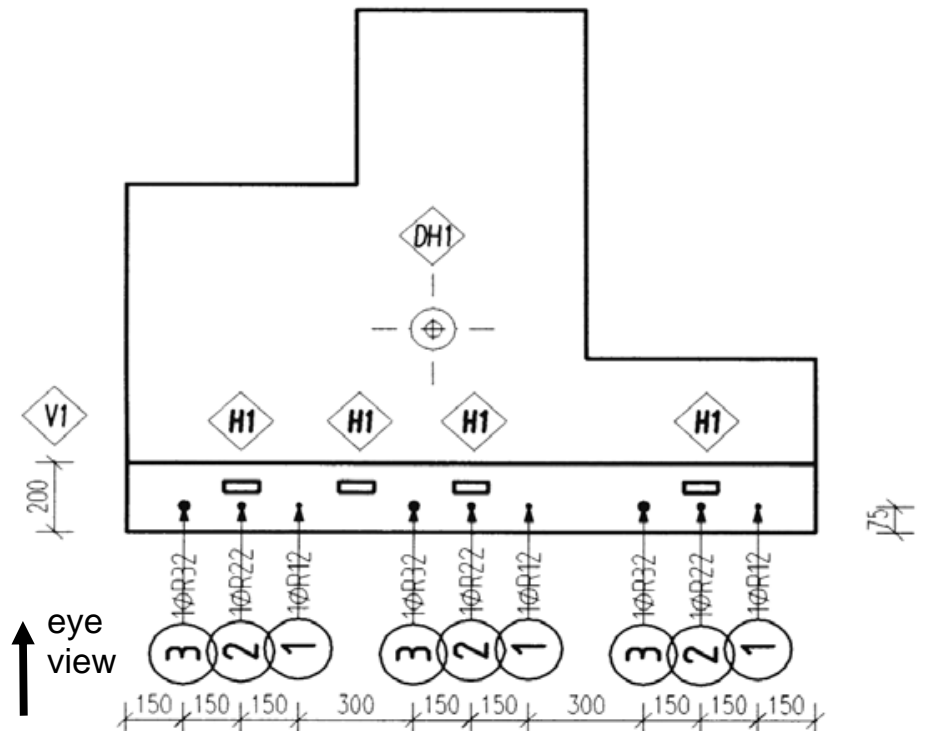

b)

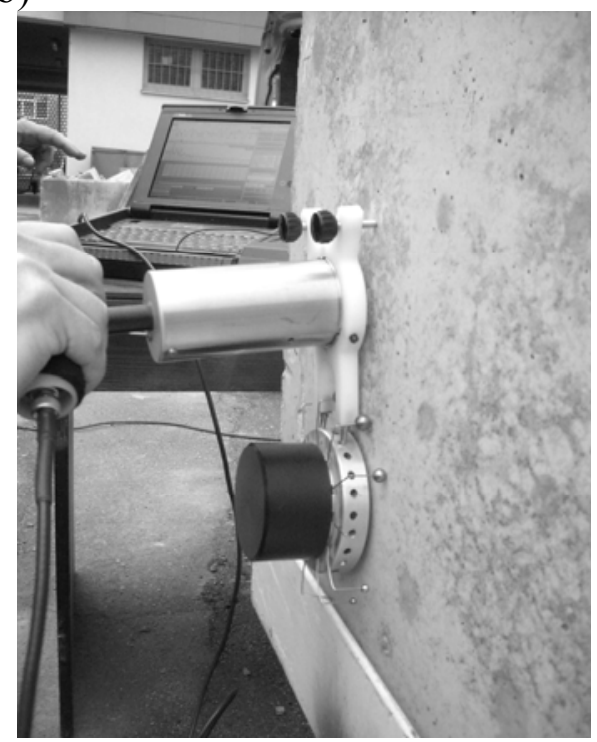

Figure 4: Schematic layout of the concrete block with artificial defects a) side view of the front wall measuring by DOCter I-E system b)

\section{CONDITIONS OF THE TESTS}

The irregular shape of the block causes multiple reflections of stress waves and a high effect of the surface wave (R-waves) on a signal and a frequency spectrum. The signals contain a high amplitude normal R-wave or a repeatable, abnormal, separated R-wave. Therefore, it was necessary to cut R-waves in every signal for further analysis. Additionally, the irregular and non-plate shape of concrete member caused multiple reflections of the P-wave. As a result, besides the fundamental mode, the additional modes of vibration are visible in the frequency spectrum. The particular modes usually are calculated on the basis of eigenvalue analysis. In the case of the tested blocks the $D / B$ ratio $(D-$ thickness of the member in impact direction, $B$ - shorter cross-sectional dimension) is higher than 0.8. According to the impact-echo principle if the $d / B$ ratio is higher than 0.9 the fundamental mode has a large amplitude and one higher than the amplitude of the next modes of vibration.

In further tests frequency distribution is characterized by the distribution of a relative frequency that can be understood as a point in the frequency domain. The frequency interval (interval between subsequent points in the frequency domain) equals $0.488 \mathrm{kHz}$ at the sampling period of $2 \mu$ s used in the tests. 
Frequency spectra for concrete blocks is similar and characterized by a sharp peak of a fundamental mode of vibration, $f_{1}$, (relative frequency $=7$ ) and successive modes: $1.71 f_{1}, 2.141 f_{1}, 2.71 f_{1}$ and $4.00 f_{1}$ (relative frequencies: $12,15,19,28$ ). In the case of the front wall of the block the fundamental frequency peak is wider and mode 2 is less visible; additionally, mode $3.00 f_{1}$ (relative frequency: 21 ) appears in the frequency spectrum.

The spectra obtained for the concrete block were used to determine the shape factor. The thickness frequency could be equal to 6 or 7 , due to the digital recording. The shape factor was calculated taking into account the signal in the centre of the front wall and the P-wave velocity measured with an ultrasonic method $(4000 \mathrm{~m} / \mathrm{s})$. The shape factors for point 6 and 7 were equal to 1.02 and 0.87 respectively. The average value of the shape factor equals 0.95 , which is close to the 0.96 typical for plate-like structures. The shape factor 0.96 was taken for further analysis.

\subsection{Detection of the steel bars}

If defects or reinforcement are present in the structure the amplitudes of particular modes should be lower, while the amplitude of frequency peak corresponding to the reflection from the defect is higher. The spectra determined for the very base of the block should be carefully examined because of a possible reflection from the steel support. The considerations above indicate that, for further analysis of frequency spectra, it is necessary to calculate expected frequencies corresponding to a reflection from particular types of artificial defects in a tested concrete block. In this case there are:

- steel bars

- defects of honeycombing types

- plastic ducts filled with cement mortar

According to the impact-echo principle, steel bars can be detected if the ratio of bar diameter to its depth is higher than 0.3. In this case only steel bars of $\varnothing 32$ and $\varnothing 22$ fulfill this requirement, and they should be visible at the relative frequency 33-35 and 25 - 27, respectively. Steel bars $\varnothing 12$ cannot be significantly detected in this situation. In table 1 the expected frequencies are shown calculated on the basis of this relationship (1).

Table 1: Expected peaks in the (relative) frequency $f$ spectrum corresponding to the reflection from steel bars, according to the distance measured on the top of the block

\begin{tabular}{|c|c|c|c|c|}
\hline \multicolumn{2}{|l|}{ Bars diameter } & $\varnothing 32$ & $\varnothing 22$ & $\varnothing 12$ \\
\hline \multicolumn{2}{|c|}{ Horizontal position in the grid } & $\mathrm{N}, \mathrm{H}$ & O-P, I-J & Q, K \\
\hline \multicolumn{2}{|c|}{ Distance from surface D [mm] } & 50 & 55 & 60 \\
\hline \multirow{2}{*}{$\begin{array}{l}\text { P wave velocity } \\
{[\mathrm{m} / \mathrm{s}]}\end{array}$} & 3800 & (33) $16 \mathrm{kHz}$ & (25) $12 \mathrm{kHz}$ & (21) $10 \mathrm{kHz}$ \\
\hline & 4000 & (35) $17 \mathrm{kHz}$ & (27) $13 \mathrm{kHz}$ & (23) $11 \mathrm{kHz}$ \\
\hline
\end{tabular}

\section{RESULTS OF I-E TESTS}

Taking into account the approximate layout of the artificial defects and armature (Fig 4a), and knowing the expected relative frequencies (Table 1), the correct answer of the stress wave propagation can be obtained. 
In figure 5 are the results of impact-echo tests carried out in the front wall of the concrete block by an impact ball of various diameters. The layout of the reinforcement in the structure can be derived transforming (relative) frequency $f$ of the highest amplitude by equation (1) onto the depth D.

a)

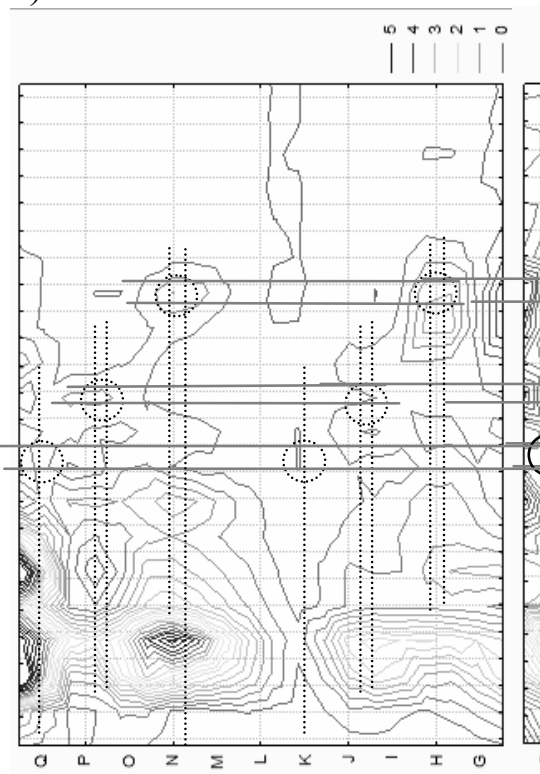

b)

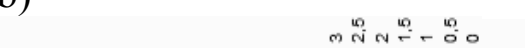

c)

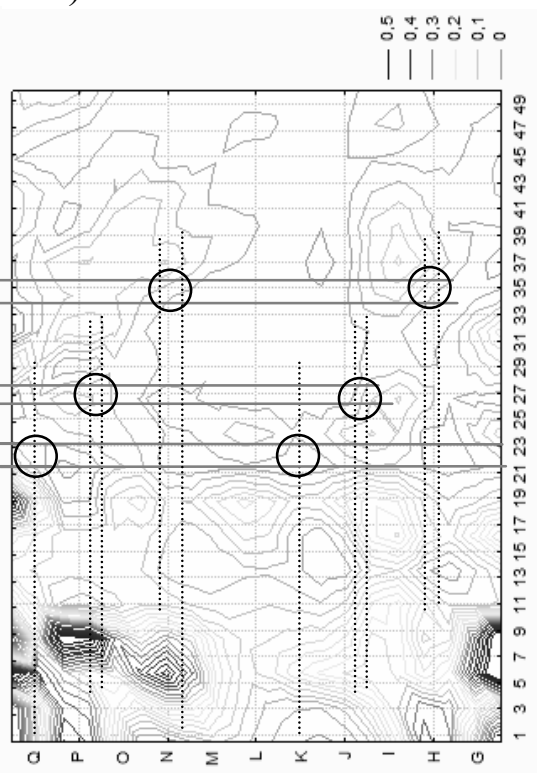

Figure 5: Relative frequency spectra (a-scan) samples composed into horizontal b-scan of the front wall of the concrete block tested by ball impactor of diameter $12 \mathrm{~mm} \mathrm{a),} 8 \mathrm{~mm} \mathrm{~b}$ ) and $5 \mathrm{~mm} \mathrm{c}$ ).

\section{CONCLUSIONS}

With a $12 \mathrm{~mm}$ impact ball a steel bar $\varnothing 32$ was detected exactly in the expected positions (50 $\mathrm{mm}$ cover) according to the velocity of P-wave $(3900 \mathrm{~m} / \mathrm{s}$ ) determined by the ultrasonic instrument before the I-E test. Reinforcement $\varnothing 22 \mathrm{~mm}$ was rather noticeable by balls of a smaller diameter $(8 \mathrm{~mm})$ at a depth of about $55 \mathrm{~mm}$. Bars $\varnothing 12 \mathrm{~mm}$ were only roughly detectable in the case of $5 \mathrm{~mm}$ ball testing, a better estimation of their position was produced by a 12 or $8 \mathrm{~mm}$ impactor. In conclusion, using a 12,8 or $5 \mathrm{~mm}$ ball it was possible to find all steel bars inserted into the massive concrete block. The approximate position of armature/defects (honeycombing, flaws, etc.), and P-wave velocity (quality of concrete) is necessary to be known before testing by the impact-echo method.

\section{ACKNOWLEDGEMENT}

This paper was arranged with the benefit of grant GA CR 103/09/1073

\section{REFERENCES}

Carino, N., J.: 1999. Nondestructive test methods. Concrete Construction Engineering Handbook (ed. E.G.Nawy), CRC Press, 19.1-19.67.

Runkiewicz, L.: 2002. Badania konstrukcji żelbetowych, Wyd. Biuro Gamma.

Sansalone, M., Street, W. B.: 1997. Impact-echo. Nondestructive evaluation of concrete and masonry, Bulbrier Press, Ithaca NY. 